\title{
AG-NGS: A powerful and user-friendly computing application for the semi-automated preparation of next-generation sequencing libraries using open liquid handling platforms
}

\section{PROTOCOL For: AG-NGS: A powerful and user-friendly computing application for the semi-automated preparation of next-generation sequencing libraries using open liquid handling platforms}

Sergio Callejas, Rebeca Álvarez, Alberto Benguria, and Ana Dopazo

Spanish National Center for Cardiovascular Research (CNIC), Madrid, Spain

BioTechniques Protocol Guide, January 2017; doi 10.2144/000114504

See full protocol online: https://benchtalk.biotechniques.com/users/7016-nathan-blow/posts/5583-protocol-for-ag-ngs-a-powerful-and-user-friendlycomputing-application-for-the-semi-automated-preparation-of-next-generation-sequencing-libraries-using-open-liquid-handling-platforms

Protocol Summary: We have developed automatic genomics NGS (AG-NGS), a computing application that allows an open liquid handling platform to be transformed into a library preparation station without losing the potential of an open platform. Implementation of AG-NGS does not require programming experience, and the application has also been designed to minimize implementation costs. Automated library preparation with AG-NGS generated high-quality libraries from different samples, demonstrating its efficiency, and all quality control parameters fell within the range of optimal values.

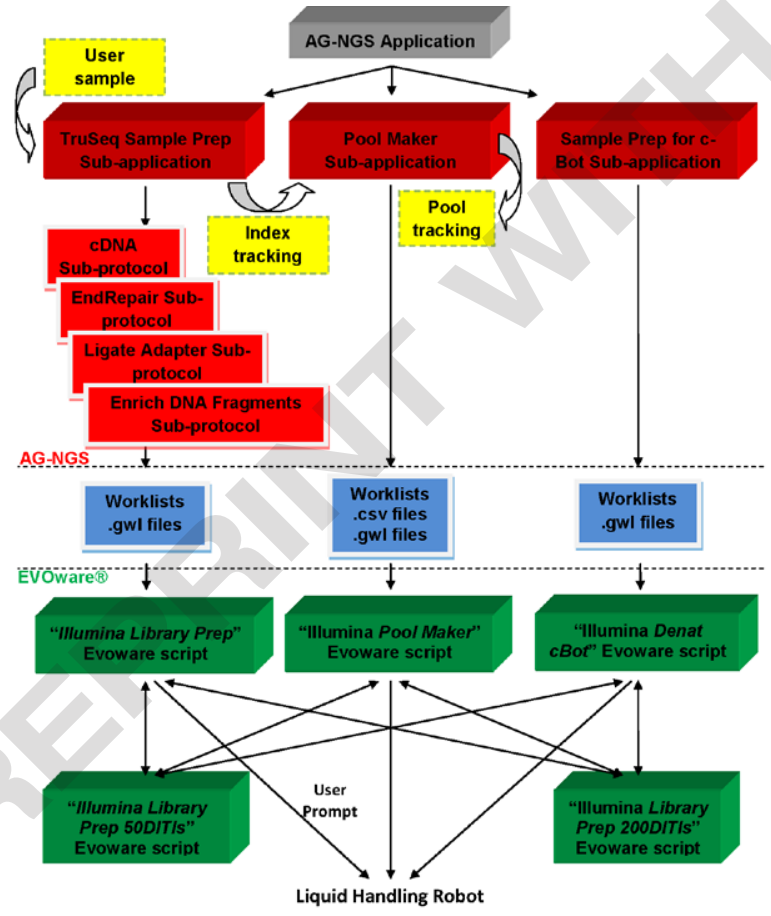

$\begin{array}{lll}\text { Application } & \text { Tracking file } \\ \text { Sub-Application } & \text { Worklist } \\ \text { Sub-Protocol } & \text { Evoware script }\end{array}$
Protocol Schematic: AG-NGS architecture. Dark red boxes show the three sub-applications included in AG-NGS application (gray) and the four sub-protocols (light red) included in the TruSeq Sample Prep sub-application. Each sub-application generates a specific group of worklists (.csv and/or.gwl files), shown in blue. Note that although TruSeq Sample Prep includes four sub-protocols, it only generates one group of worklists. Files related to sample tracking are shown in yellow. The user sample name file is generated by the user and imported into TruSeq Sample Prep, which will automatically generate an index-tracking file containing the sample names and associated index numbers. This file has to be imported into the Pool Maker sub-application before samples can be pooled. Pool Maker then generates an output file (pool tracking file) so that each sample can be easily tracked through the library preparation process. Once worklists have been generated, they are executed by different EVOware scripts (green boxes), one for each AG-NGS sub-application. Note that the lllumina Library Prep EVOware script is able to execute worklists from four different sub-protocols; for this reason the user will be asked which of the four sub-protocols he or she wishes to execute. The EVOware scripts Illumina Library Prep 50DITIs and Illumina Library Prep 200DITIs do not execute any worklists but instead interact with the other three scripts to prevent the robot from pausing the script when all the tip racks have been used. If tips have not been replaced during the run, EVOware will display an error message, and the robot will pause. Should this happen, simply refill the tip racks and resume the script. 